\title{
Candidates for thrombolytic treatment in acute ischaemic stroke-where are our patients in Hong Kong?
}

\author{
Y C A Siu, T W Wong, C C Lau
}

\begin{abstract}
Objective-Tissue plasminogen activator (t-PA) has been approved by the Food and Drug Administration in the treatment of patients with acute ischaemic stroke presenting within three hours from onset of symptoms. This study aims to identify the potential number of stroke patients suitable for t-PA in Hong Kong.

Methods-All patients with a clinical diagnosis of acute stroke were recruited. Data collected included demographics, vital signs, medical history, contraindications to thrombolysis, severity of stroke (Canadian neurological scale), time course from onset of symptoms to computed tomography, computed tomography results, and final diagnoses by physicians. Results-During the five month study period, 201 patients were recruited and nine were subsequently excluded from further analysis because computed tomography was not performed. Their mean age was 70.9 (range from 41-91) years. Eighty (41.7\%) and $100(52.1 \%)$ patients presented to our emergency department within two hours and three hours respectively from symptom onset. The mean severity score (Canadian neurological scale) was 7.83 (out of a maximum of 11.5). A total of $132(68.8 \%)$ patients had acute ischaemic stroke confirmed by computed tomography. Mean delay in computed tomography was 4.91 hours. Fourteen $(7.3 \%)$ and $52(27.1 \%)$ of all patients had computed tomography of the brain done within one and two hours respectively. Only 20 patients $(10.45 \%)$ could meet the three hour criteria as stated in the National Institute of Neurologic Disorders and Stroke rt-PA stroke study and seven $(3.6 \%)$ of them were confirmed to have acute ischaemic stroke. Two patients were further excluded because of high systolic blood pressure and current warfarin medication.

Conclusion-At present very few patients could benefit from thrombolytic treatment. Delays in the chain of recovery in stroke management should be identified and corrected.

(F Accid Emerg Med 1999;16:412-417)
\end{abstract}

Keywords: acute ischaemic stroke; delay; thrombolysis

Correspondence to:

Dr Siu Yuet-chung Axel,

Medical and Health Officer

(e-mail: axel@hknet.com).

Accepted 17 July 1999

The use of tissue plasminogen activator (t-PA) in the management of stroke has been approved by the Food and Drug Administration since the publication of the National Institute of Neurologic Disorders and Stroke rt-PA stroke (NINDS) study. ${ }^{12}$ However, it has been shown that a better neurological outcome is restricted only to patients who can receive t-PA within three hours from onset of symptoms. According to a meta-analysis with 1297 patients, there was about $6.5 \%$ absolute reduction in poor outcome, which was defined as death or dependency in daily activities. ${ }^{3}$ The potential benefit of the drug is greatly limited by the narrow therapeutic window.

There are some studies published after the NINDS study to investigate the proportion of patients who may be eligible for thrombolytic treatment. $^{4-6}$ Though t-PA has not been adopted in the treatment of acute ischaemic stroke in Hong Kong, we would like to investigate the potential for its use in the region. We would also like to identify the sources of delay from symptom onset to examination by computed tomography in the hospital.

\section{Methods}

STUDY SETTING

Acute stroke is the third commonest cause of death in Hong Kong. ${ }^{7}$ It is one of the commonest medical problems encountered in our department. In Hong Kong, most patients with acute stroke will be admitted to regional public hospitals. As in the UK patients call an ambulance by dialling " 999 " for the emergency services and an ambulance will arrive in less than 20 minutes in most circumstances.

Our hospital is a regional hospital serving a population of 600000 in the eastern part of Hong Kong Island. At the time of the study, we were seeing around 450 patients a day on average. Patients are triaged by a nurse into four major categories: emergency, urgent, semiurgent, and non-urgent. Emergency cases are seen immediately and urgent ones are seen within 30 minutes. In our department, all patients with a provisional diagnosis of acute stroke will be admitted to the medical ward. There is no direct referral for computed tomography in our department. The decision for requesting computed tomography of the brain will be left to the ward physicians. The waiting time will depend on the severity of symptoms and the patient load. Stable stroke patients may need to wait up to several hours for computed tomography of the brain.
STUDY METHOD

This was a prospective study of consecutive cases. All patients admitted to our department 


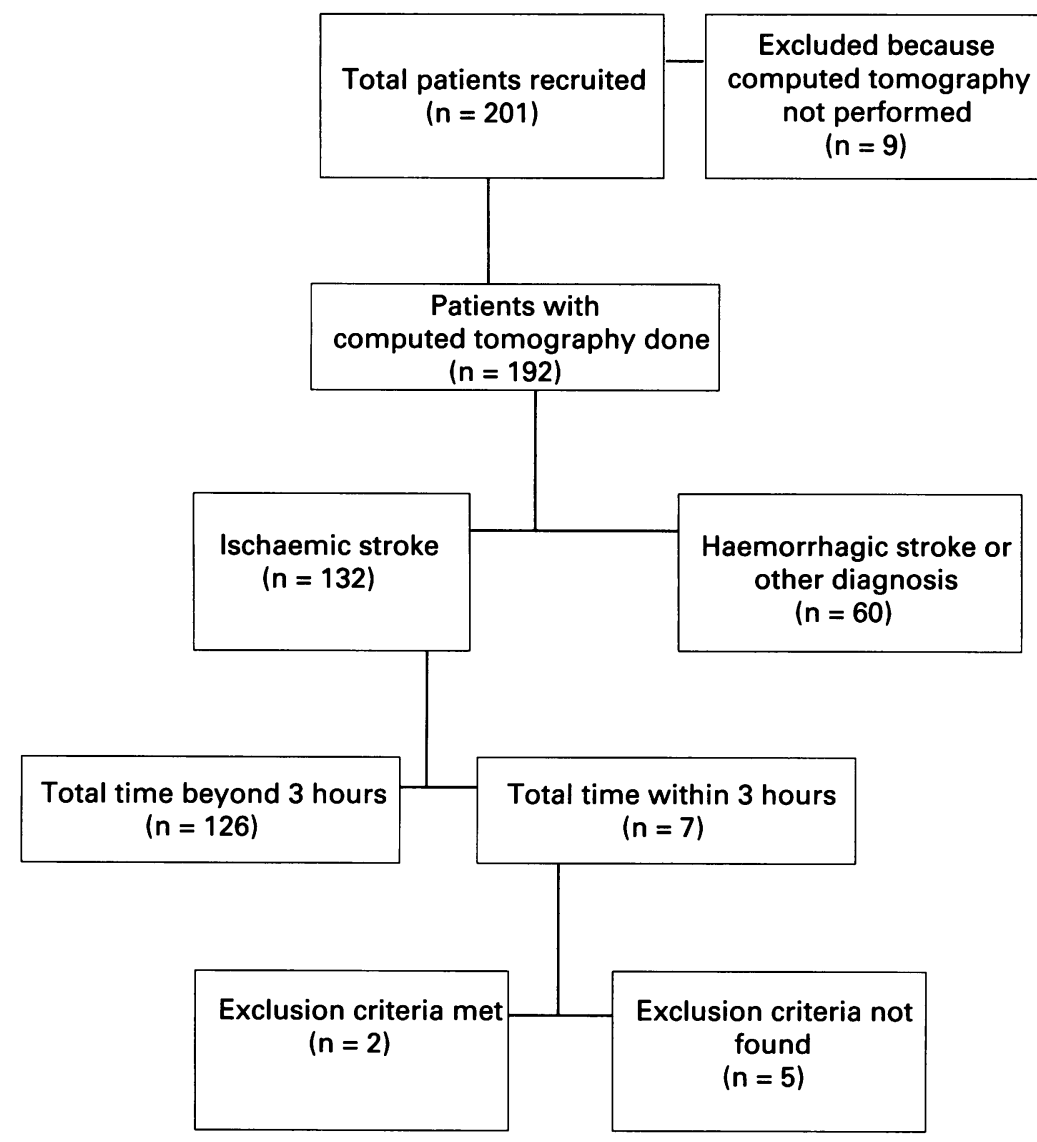

Figure 1 Flow chart for the selection of patients according to the diagnosis and the time limit. hours". The time of ambulance arrival to the scene was also obtained from the ambulance run sheet. The time when the patient arrived at our emergency department, the doctor contact time, and the time when the patient was transferred out were recorded in the patient's medical records. The time when brain scan was performed was retrieved from the computer record of the radiology department.

The descriptive and analytical statistics were performed with SPSS PC statistical package. Multivariate analysis with logistic regression was used to determine the possible association between the success in meeting the time criteria and the demographic data, history of stroke or transient ischaemic attack, severity of stroke, transfer method to the emergency department, and the different time intervals. They were expressed in terms of odds ratio (OR) and a $p$ value of 0.05 was used as the significant level. Similar methods and variables have been used in a previous study. ${ }^{9}$

\section{Results}

During the five months from 25 July 1997 to 24 December 1997, there were 201 patients with a clinical diagnosis of acute stroke admitted to the accident and emergency department. Only 192 of them underwent computed tomography of the brain and the rest were considered not to have clinical stroke by the admitting physician and they were excluded from further analysis. There were 105 male and 87 female patients. Their ages ranged from 41 to 91 (mean age 70.9 ) years. Hypertension was found in 90 patients $(46.9 \%)$ and was the commonest risk factor, followed by previous cerebrovascular accident $(52 / 27.1 \%)$ and smoking (45/23.4\%). The prevalence of atrial fibrillation was low (11/5.7\%) (table 1).

Hemiplegia was the commonest presenting symptom (146/76.1\%); 75 (39.1\%) were left sided and $71(37.0 \%)$ right sided. Dysphasia was found in $49(25.5 \%)$ patients, $39(20.3 \%)$ presented with a change in conscious level, and $29(15.1 \%)$ suffered from dizziness or vertigo.

The majority of patients were stable and had a high score on the Canadian neurological scale $(118 / 192$ had a score $\geqslant 8.5)$ and the Glasgow coma score (163/192 had scores of at least 13). The mean score in the Canadian neurological scale was $7.83 ; 100(69.8 \%)$ of the patients were assessed as urgent or above by the triage nurse.

Table 1 Medical history of the 192 patients; there were 105 males and 87 females with a mean (range) age of 70.9 (41-91) years

\begin{tabular}{lll}
\hline Patient characteristics & Present & Absent \\
\hline Past medical health & & \\
Hypertension & 90 & 102 \\
Diabetes mellitus & 35 & 157 \\
Old cerebrovascular accident & 52 & 140 \\
Transient ischaemic attack & 7 & 185 \\
Atrial fibrillation & 11 & 181 \\
Ischaemic heart disease & 28 & 164 \\
Congestive heart failure & 7 & 185 \\
Smoking & 45 & 147 \\
Major contraindication to thrombolysis & & \\
Recent gastrointestinal bleeding & 3 & 189 \\
Recent major operation & 1 & 191 \\
Bleeding tendency & 7 & 185 \\
\hline
\end{tabular}
the ambulance control if the emergency ambulance service was called. If the time interval was greater than 24 hours, it would be categorised into the group of "equal or greater than 24

The time intervals from the onset of symptoms to the time of computed tomography were documented. The time of onset of stroke was defined as the time when the symptoms were noticed by either the patient or the witness. The time of decision made to seek medical help was defined as the time when the patient or the witness decided a medical consultation was required. It depended either on their recall or according to the record from 
Table 2 Summary of the time courses from symptom onset to computed tomography of brain $(n=192)$

\begin{tabular}{llll}
\hline & $\begin{array}{l}\text { Mean time } \\
(\text { min })\end{array}$ & $\begin{array}{l}\text { Median time } \\
(\text { min })\end{array}$ & $\begin{array}{l}\text { Interquartile range } \\
(\text { min })\end{array}$ \\
\hline Symptom onset to decision made to seek medical help & $\mathrm{N} / \mathrm{A}$ & 174.50 & $5.00-873.75$ \\
$\star$ Decision made to arrival at emergency department $(\mathrm{n}=79)$ & 134.54 & 55.0 & $37.0-118.00$ \\
†Decision made to ambulance arrival at scene $(\mathrm{n}=113)$ & 12.15 & 10.00 & $7.00-13.00$ \\
tAmbulance arrival to emergency department arrival $(\mathrm{n}=113)$ & 18.88 & 17.00 & $12.00-22.50$ \\
Contact time by medical staff & 14.89 & 10.00 & $6.00-19.00$ \\
Assessment time in emergency department & 35.48 & 26.00 & $17.00-42.00$ \\
Computed tomography waiting time & 294.85 & 227.50 & $113.00-380.75$ \\
\hline
\end{tabular}

*For patients who did not take ambulance to emergency department.

†For patients who took ambulance to emergency department.

N/A = Not applicable.

Ischaemic stroke was the major cause of stroke among our patients (132/68.8\%) whereas haemorrhagic stroke was only found in $38(19.8 \%)$ patients. Patients or their relatives initiated the decision to seek medical help in most of the incidents $(160 / 83.3 \%)$ and $113(58.9 \%)$ of the patients were transferred to our department by ambulance.

Among the 132 patients with acute ischaemic stroke, only seven $(3.6 \%)$ could have computed tomography done within three hours from symptom onset. The cut off point was set at three hours with reference to the NINDS study. If the limit was extended to six hours, a total of 23 patients $(12.0 \%)$ could meet the criteria. However, two patients did not meet the criteria for thrombolysis. One had systolic blood pressure greater than $185 \mathrm{~mm} \mathrm{Hg}$ and the other was taking warfarin for chronic rheumatic heart disease. Only five patients $(2.6 \%)$ remained eligible as potential candidates for thrombolysis.

Eighty of the 192 patients arrived at our emergency department within two hours; this rose to 100 if a three hour cut off was used. The mean time interval for the ambulance to arrive at the scene was $12.15 \mathrm{~min}$ and the mean time interval to take the patient to the emergency department was $18.88 \mathrm{~min}$. The mean waiting time for doctor assessment was

Table 3 Distribution of computed tomography waiting time

\begin{tabular}{llllllll}
\hline & $<30 \mathrm{~min}$ & $30-60 \mathrm{~min}$ & $1-3$ hours & $3-6$ hours & $6-12$ hours & $12-24$ hours & $>24$ hours \\
\hline No (\%) & $7(3.6)$ & $7(3.6)$ & $65(33.9)$ & $61(31.8)$ & $43(22.4)$ & $8(4.2)$ & $1(0.5)$ \\
\hline
\end{tabular}

Table 4 Logistic regression analysis of eligibility using three hour time limit

\begin{tabular}{|c|c|c|}
\hline & $O R$ & p Value \\
\hline \multicolumn{3}{|l|}{ Patient factor } \\
\hline Age $>65$ & 0.43 & 0.201 \\
\hline Sex $=$ male & 1.38 & 0.595 \\
\hline Previous history of stroke & 1.00 & 0.997 \\
\hline Previous history of transient ischaemic attack & 0.33 & 0.559 \\
\hline \multicolumn{3}{|l|}{ Presenting symptoms } \\
\hline Hemiplegia & 0.72 & 0.646 \\
\hline Dysphasia & 1.46 & 0.472 \\
\hline Altered mental state & 2.66 & 0.172 \\
\hline Dizziness/vertigo & 3.89 & 0.312 \\
\hline \multicolumn{3}{|l|}{ Severity of stroke } \\
\hline Glasgow coma score $<13$ & 1.30 & 0.688 \\
\hline Canadian neurological scale $<8.5$ & 5.30 & 0.128 \\
\hline \multicolumn{3}{|l|}{ Transfer method } \\
\hline By ambulance & 1.75 & 0.521 \\
\hline \multicolumn{3}{|l|}{ Triage category } \\
\hline Category 1 or 2 & 0.59 & 0.616 \\
\hline \multicolumn{3}{|l|}{ Time intervals } \\
\hline Time from onset to decision made $<15 \mathrm{~min}$ & 10.62 & 0.042 \\
\hline Time from decision made to arrival at emergency & & \\
\hline department $<60 \mathrm{~min}$ & 63.59 & 0.865 \\
\hline Medical officer contact time $<15 \mathrm{~min}$ & 0.44 & 0.589 \\
\hline Time from assessment to disposal $<60 \mathrm{~min}$ & 153.11 & 0.891 \\
\hline Computed tomography time $<60 \mathrm{~min}$ & 23.26 & 0.011 \\
\hline
\end{tabular}

14.89 (range 0-86) min and the mean duration of stay in the emergency department after assessment was 35.48 (range 8-645) $\mathrm{min}$. One patient had an extremely long stay time (645 $\mathrm{min}$ ) because the diagnosis was initially missed by a junior doctor and subsequently identified by a senior doctor in the observation ward. Only $14(7.3 \%)$ and $52(27 \%)$ of the patients could have computed tomography of the brain performed within one and two hours, respectively, after admission. The mean waiting time for the scan was 4.91 hours (tables 2 and 3).

The potential factors that might affect whether patients could have computed tomography confirmation of diagnosis within three hours from symptom onset were analysed with logistic regression (table 4). A delay of less than 15 minutes from onset of symptoms to the decision to seek medical help $(O R=10.62, p=$ 0.042 ) and a computed tomography waiting time of less than one hour from admission (OR $=23.26, \mathrm{p}=0.011$ ) were the only two significant factors predicting which patients could meet the time limit. Sex and age greater than 65 were not shown to be significant predictors. None of the presenting symptoms showed any value as predictors of delay. A previous cerebrovascular accident or transient ischaemic attack also did not shorten the arrival time. The severity of stroke in terms of Canadian neurological scale and Glasgow coma score were not proved to be significant predictors. A higher triage category (category 2 or above) was not found to significantly shorten the delay.

\section{Discussion}

In our series, only a minority of patients were eligible as potential candidates for thrombolysis. Our results were worse than most of the overseas studies of a similar nature. Zweifler $e t$ al found that about $36 \%$ of their patients were eligible for t-PA but only $19 \%$ of these patients received the treatment. ${ }^{6}$ The proportion of patients $(52 \%, 100 / 192)$ who arrived at our emergency department within three hours from symptom onset was similar to a supplementary study to the NINDS study, which found that $39 \%$ of acute stroke patients arrived at the emergency department within $90 \mathrm{~min}$ and $20 \%$ at $91-180$ min. ${ }^{4}$ Early arrival was related to the use of an ambulance, stroke at daytime, and stroke when the patient was at work. A study of 96 patients by Feldmann et al showed that about half of the patients presented within four hours and a sudden onset of stable deficit was the unique independent 
factor associated with early presentation. ${ }^{10}$ Similar study by Azzimondi et al found that 59 out of 189 patients arrived within two hours and 100 patients arrived within five hours. ${ }^{5}$ Onset when the patient was awake, an altered mental state, and limb paralysis were the only independent predictors of early arrival. A study in Leicestershire also attempted to address the possible factors causing delay. ${ }^{11}$ The delay was mainly related to admission through the bed allocation bureau, patients living alone, and stroke onset at night. In our study, making a decision to seek medical help within 15 minutes from onset was one of the significant factors to predict early presentation. We empirically chose 15 minutes, as we believed it was a reasonable time interval to let the witness or patient to call for help if they thought it was serious. All other factors relating to the mode of presentation or transfer were not found to be statistically significant. We did not look into where and when the stroke happened. A similar study in the United States, which also used logistic regression, found that the use of emergency medical services and being white increased the odds of early presentation to the emergency department. ${ }^{9}$

A previous study in Hong Kong showed that $63 \%$ of patients arrived at hospital within 12 hours after a stroke. ${ }^{12}$ We have achieved a similar result with $70.6 \%$ of patients arriving at our hospital within 12 hours from onset. Their results also showed a higher tendency for patients with haemorrhagic stroke to present earlier. It may be related to the higher chance of experiencing severe symptoms, for example altered mental state in haemorrhagic stroke. However, we could not confirm the significance of this symptom in our logistic regression analysis.

\section{IMPLICATIONS OF OUR STUDY}

We showed that about $69 \%$ of our patients had strokes that were ischaemic in origin and would be potential candidates for thrombolysis; however, only a few were eligible and the major reason was delay. The delay was mainly caused by late presentation to the emergency department and delay in obtaining a computed tomography scan. In fact, delay in presentation to the emergency department was the commonest reason for ineligibility. ${ }^{13}$ In order to allow more patients to benefit from thrombolytic treatment, we should understand the current concept of the chain of recovery in stroke.

CHAIN OF RECOVERY IN STROKE

In the past, treatment of stroke was mainly supportive and aimed to prevent further complications. This was changed after the implementation of thrombolytic treatment and a chain of recovery for stroke was suggested similar to the chain of survival in cardiac arrest. ${ }^{14}$ The chain involves the early identification of symptoms of stroke by the patient or bystanders, dispatch of specific pre-hospital life support services, rapid access and transport by emergency medical service personnel to the receiving hospital, and the notification of a special team that can coordinate the specific investigation and intervention for stroke patient: the stroke team.

\section{EARLY IDENTIFICATION OF STROKE SYMPTOMS}

Obviously, decreasing the delay in recognition of stroke symptoms is very important to meet the short therapeutic window. Educating the public and patients at risk of "brain attack" of the potential benefits of early presentation may be helpful. Members of the public were generally not aware of their own risk for stroke. Samsa et al found that only $41 \%$ of their respondents noticed their increased risk for stroke. ${ }^{15}$ Most of them recalled that they had been informed by a physician. Younger patients, depressed patients, patients with poor general health, and those with a history of transient ischaemic attacks were more likely to be aware of their stroke risk. In a study published in 1997, the authors found that only one fourth of patients could correctly interpret their symptoms as stroke. ${ }^{16} \mathrm{~A}$ majority of the late presenters thought that their symptoms were not serious. A more recent survey on public knowledge of stroke warning signs and risk factors found that $57 \%$ of the 1066 respondents from telephone interviews could identify at least one stroke warning sign and $68 \%$ could list at least one risk factor of stroke. ${ }^{17}$ Only $57 \%$ of those respondents with a history of hypertension knew that hypertension was a risk factor for stroke. Respondents with a history of smoking and diabetes mellitus were even worse as only $35 \%$ and $13 \%$ of them could identify their own risk. Public education is essential to reduce the delay. Alberts et al started a multifaceted programme of public and professional education that yielded a $49 \%$ increase in the number of acute ischaemic stroke patients who presented within 24 hours from symptom onset. ${ }^{18}$ Delay in making the decision to seek medical help was one of the major causes of ineligibility in our study. However, we have not explored the reasons for the late decision. Further studies are required to address this issue and to develop local strategies of public education to encourage early presentation to the emergency department.

\section{PRE-HOSPITAL PHASE}

Many studies have found ambulance transfer to facilitate early arrival. ${ }^{416} \mathrm{~A}$ local study showed that the mean time interval for the ambulance staffed by emergency medical assistants, who have intermediate level training, to arrive at the scene was $6.99 \mathrm{~min}$ and at the patient's side was 9.07 min. ${ }^{19}$ The mean time the ambulance crews spent at the patient's side was about $12 \mathrm{~min}$ and the travel time to hospital was about $11 \mathrm{~min}$. Our results were comparable with the results of that study. Educating ambulance staff on the concept of "brain attack" may help to expedite the transfer process. Though patient transfer by the emergency services was shown to be associated with early presentation, we could not demonstrate its association with the success to meet the time criteria. ${ }^{10}$ Since the main delay is in patients' delay in seeking medical attention, the 
impact of quicker ambulance transfer may not be that great.

An accurate pre-hospital diagnosis by paramedics is also another important element that may affect the delay. A review of 86 cases with a pre-hospital diagnosis of stroke or transient ischaemic attack showed that the emergency medical service dispatcher and the paramedics could correctly identify $52 \%$ and $72 \%$ of cases respectively. ${ }^{20}$ Patients requiring advanced life support by paramedics could be seen by physicians and undergo computed tomography earlier. In another study of 81 patients, the sensitivity of paramedic diagnosis of stroke was $61 \%$ and the positive predictive value was $77 \%$. $^{21}$

PROMPT DIAGNOSIS IN EMERGENCY DEPARTMENT In the emergency department phase, accuracy of nurse triage is another important issue. In our series, $58(30.2 \%)$ of our patients were categorised as semiurgent at triage; this may result in prolonged waiting time in the emergency department. Under-triage will definitely cause a certain degree of delay. The triage guidelines may need to be revised to facilitate earlier medical attention. The other issue is the accuracy of the clinical diagnosis of stroke made by emergency physicians. Kothari et al found that emergency physicians made an incorrect diagnosis of stroke or transient ischaemic attack in 19 out of 365 (5\%) patients. ${ }^{22}$ In our 201 patients with a clinical diagnosis of acute stroke, $22(10.4 \%)$ patients had a final diagnosis other than acute stroke. Lack of computed tomography support in the emergency department made it more difficulty for the attending doctor to reach the correct diagnosis. Various conditions could mimic acute stroke. Libman et al suggested that decreased conscious level would increase the odds and history of angina would decrease the odds of "stroke mimic" conditions in a multivariate analysis. ${ }^{23} \mathrm{We}$ do not know how many stroke cases were initially missed in the emergency department and were subsequently identified by the physicians in our study. In order to enhance the coordination and reduce the delay after arrival, some centres have established a stroke team to coordinate the investigation and subsequent thrombolysis. ${ }^{24}$ However, very few hospital emergency departments had their own protocol for thrombolytic treatment in acute ischaemic stroke. ${ }^{25} \mathrm{~A}$ consensus should be established among emergency physicians and neurologists on the use of t-PA in acute stroke.

EARLY COMPUTED TOMOGRAPHY AND ACCURATE INTERPRETATION

In our study it was found that performing computed tomography of the brain within an hour after admission was predictive of meeting the time limit. However, only $7.2 \%$ of our patients could meet this criteria (table 4). The median time was $227.5 \mathrm{~min}$, which was much longer than the $72 \mathrm{~min}$ reported in a similar study in the United States. ${ }^{9}$ The delay in getting a scan was mainly for administrative reasons. Because of the great demand for computed tomography, the service is usually available only for critically ill patients. Stable patients usually have to wait for several hours. The problem of delay was also observed in overseas centres. Aziz et al reported that there was a reduction in time interval from symptom onset to arrival at the emergency department after the approval of t-PA in the management of acute stroke. However, the door to computed tomography time did not change much and was around two hours on average. ${ }^{26}$ If thrombolysis is to be implemented for treatment of acute ischaemic stroke in Hong Kong, a direct referral for computed tomography examination from the emergency department is required to shorten the delay.

According to the NINDS study, patients with intracranial haemorrhage will be excluded from thrombolysis. The doctor who interprets the computed tomogram will make the final decision and the accuracy will depend on the capability of the interpreter. A study to evaluate accuracy in interpretation found that the accuracy of emergency physicians was only $67 \%$ whereas neurologists and radiologists achieved $83 \% .^{27} \mathrm{~A}$ radiologist should also be available to interpret the film without delay to avoid inappropriate administration of a thrombolytic agent.

CONTROVERSIES IN THROMBOLYTIC TREATMENT $t-P A$ - to use it or not?

Though the Food and Drug Administration has approved the clinical use of t-PA for acute ischaemic stroke, there is still debate about it. The NINDS group reported $11 \%-13 \%$ absolute increase in favourable outcome at three months in the group receiving t-PA. ${ }^{1}$ The effect was not limited by age and stroke subtypes. There was a roughly 10 -fold increase in symptomatic intracerebral haemorrhage $(6.4 \% v$ $0.6 \%$ ) in the treatment group, though the mortality rate was not proved to be significantly different.

Some authors believed that the benefit reported in the NINDS study was too generous and the benefit was not equally distributed among various causes of ischaemic stroke. ${ }^{28}$ The only established effective use was to lyse acute thromboemboli. Furlan and Kanoti also had queries about the preciseness of subgroup analysis in the original study. ${ }^{29} \mathrm{On}$ the other hand, there were other authors who insisted that thrombolytic treatment should be the first line treatment for those who could fulfil the criteria. ${ }^{30}$ This controversy will continue to be hotly debated until further large scale studies can bring us the definitive answer.

\section{Margin of benefit for mild symptoms}

The role of thrombolytic treatment in patients with mild symptoms is unclear. All patients with mild symptoms were excluded from the European Cooperative Acute Stroke Study. ${ }^{31}$ The NINDS study recruited patients with different severity. ${ }^{1}$ However, there was no detailed description on the extent the group with mild symptoms improved with t-PA. The risk of intracerebral haemorrhage needed to be balanced with the extent of benefit from the treatment. Our patients belonged mainly to the group with mild symptoms. More than $60 \%$ of 
them had a score of at least 8.5 on the Canadian neurological scale. If the benefit is confined only to severe cases, the application of thrombolytic treatment in our locality will be limited. The extent of benefit of thrombolytic treatment in patients with mild symptoms needs to be addressed with further studies.

LIMITATIONS OF THE STUDY

The major limitation of our study is the dependence on patient or witness recall for the pre-hospital time intervals. The inaccuracy in memory may be a major contribution to the great variability of data. If thrombolysis is implemented in Hong Kong, there will be a strong need to make sure that the time of onset is accurately ascertained so as to avoid the catastrophic side effects.

\section{Conclusions}

With the current setting, the number of stroke patients eligible for thrombolysis is very small. To increase the number of potential candidates for thrombolysis, major efforts to educate the public are needed to decrease pre-hospital delay. Also, arrangement must also be made to shorten the delay in performing computed tomography and a stroke team should be established to coordinate patient care.

The authors would like to thank the Ambulance Service (Eastern Hong Kong Division), and all the medical and nursing staf orn the Accident and Emergency Department, Pamela Youde Nethersole Eastern Hospital for their support in the project. Conflict of interest: none.

Funding: none.

1 National Institute of Neurologic Disorders and Stroke rt-PA Stroke Study Group. Tissue plasminogen activator for acute ischaemic stroke. N Engl $f$ Med 1995;333:1581-7.

2 Food and Drug Administration. Newsletter. Washington, DC: FDA, 18 January 1996.

3 Wardlaw JM, Warlow CP, Counsell C. Systematic review of evidence on thrombolytic therapy for acute ischaemic evidence on thrombolytic the

4 Barsan WG, Brott TG, Broderick JP, et al. Time of hospital presentation in patients with acute stroke. Arch Intern Med 1993;153:2558-61.

5 Azzimondi G, Bassein L, Fiorani L, et al. Variables associated with hospital arrival time after stroke: effect of delay on the clinical efficiency of early treatment. Stroke 1997;28:537-42.

6 Zweifler RM, Brody ML, Graves GC, et al. Intravenous t-PA for acute ischaemic stroke: therapeutic yield of a stroke code system. Neurology 1998;50:501-3.

7 Department of Health, Hong Kong Government. Annual report (1994/5). Hong Kong: Hong Kong Government, $1995: 51$.

8 Cote R, Battista RN, Wolfson C, et al. The Canadian neurological scale: validation and reliability assessment. Neurology 1989;39:638-43.
9 Kothari R, Jauch E, Broderick J, et al. Acute stroke: delays to presentation and emergency department evaluation. Ann Emerg Med 1999;33:3-8.

10 Feldmann E, Gordon N, Brooks JM, et al. Factors associated with early presentation of acute stroke. Stroke 1993;24:1805-10.

11 Harper GD, Haigh RA, Potter JF, et al. Factors delaying hospital admission after stroke in Leicestershire. Stroke 1992;23:835-8.

12 Kay R, Woo J, Poon WS. Hospital arrival time after onset of stroke. $\mathcal{F}$ Neurol Nerurosurg Psychiatry 1992;55:973-4

13 O'Connor R, McGraw P, Edelsohn L. Thrombolytic therapy for acute ischaemic stroke: why the majority of patients remain ineligible for treatment. Ann Emerg Med 1999;33:9-14.

14 Pepe PE, Zachariah BS, Sayre MR, et al. Ensuring the chain of recovery for stroke in your community. Acad Emerg Med 1998;5:352-8.

15 Samsa GP, Cohen SJ, Goldstein LB, et al. Knowledge of risk among patients at increased risk for stroke. Stroke 1997;28: among pacic

16 Williams LS, Bruno A, Rouch D, et al. Stroke patients knowledge of stroke: influence on time of presentation. Stroke 1997;28:912-15.

17 Pancioli AM, Broderick J, Kothari R, et al. Public perception of stroke warning signs and knowledge of potential risk factors. $\mathcal{F} A M A$ 1998;279:1288-92.

18 Alberts MJ, Perry A, Dawson DV, et al. Effects of public and professional education on reducing the delay in presentation and referral of stroke patients. Stroke 1992;23:352-6.

19 Wong TW, Lau CC, Pang KC. A review of prehospital care provided by ambulance attendants with extended training in Hong Kong. Hong Kong fournal of Emergency Medicine 1996;3:13-18.

20 Kothari R, Barsan W, Bott T, et al. Frequency and accuracy of prehospital diagnosis of acute stroke. Stroke 1995;26: 937-41.

21 Smith WS, Isaacs $M$, Corry MD. Accuracy of paramedic identification of stroke and transient ischemic attack in the field. Prehospital Emergency Care 1998;2:170-5.

22 Kothari R, Brott T, Broderick JP, et al. Emergency physicians: accuracy in the diagnosis of stroke. Stroke 1995; physicians:

23 Libman RB, Wirkowski E, Alvir J, et al. Conditions that mimic stroke in the emergency department. Implication for acute stroke trials. Arch Neurol 1995;52:1119-22.

24 Huff JS. Acute stroke intervention: where are the patients? (abstract). Acad Emerg Med 1997;4:373.

25 Heller MB, Kunnel B, Arcona S. Thrombolytics and stroke: what do emergency medicine residents perceive? (abstract). Ann Emerg Med 1998;32:S32.

26 Aziz N, Williams SR, Williams CM, et al. The effect of FDA approval of thrombolytic therapy on the management of acute stroke in the emergency department (abstract). Ann Emerg Med 1998;32:S42.

27 Schriger DL, Kalafut M, Sidney S, et al. Cranial computed tomography in acute stroke. Physician accuracy in determining eligibility for thrombolytic therapy. $\mathfrak{F} A M A$ 1998;279:1293-7.

28 Caplan LR, Mohr JP, Kistler JP, et al. Should thrombolytic therapy be the first-line treatment for acute ischaemic stroke? Thrombolysis-not a panacea for ischaemic stroke. stroke? Thrombolysis-not a panacea for

29 Furlan AJ, Kanoti G. When is thrombolysis justified in patients with acute ischaemic stroke? A bioethical perspective. Stroke 1997;28:214-18.

30 Grotta J. Should thrombolytic therapy be the first-line treatment for acute ischemic stroke? tPA-the best current option for most patients. $N$ Engl f Med 1997;337:1310-12, 1313.

31 European Cooperative Acute Stroke Study (ECASS). Intravenous thrombolyisis with recombinant tissue plasminogen venous thrombolyisis with recombinant tissue plasminogen 1017-25. 